\title{
SECONDARY ELECTRON YIELD OF A THIN FILM COATING ON THE APS RF CAVITY TUNERS*
}

\author{
D.R. Walters ${ }^{\dagger}$ and Q. Ma \\ Advanced Photon Source, Argonne National Laboratory, Argonne, Illinois
}

\begin{abstract}
A thin film coating of titanium nitride was applied to the surface of $\mathrm{rf}$ cavity tuners to reduce secondary electron generated when the existing copper base material is in place. The tuners are used in high-field gradients, where electron heating of the tuner is a problem. By reducing the generation of electrons the temperature of the tuners will be reduced, for better reliability. This paper compares characteristics useful for rf cavity tuners, such as surface composition and secondary electron yield, for copper, titanium-coated copper, and titanium-nitridecoated copper. The process and the setup of equipment used to apply the coatings are summarized.
\end{abstract}

\section{INTRODUCTION}

The Advanced Photon Source (APS) is a facility for material science research. It provides users with a source of $\mathrm{x}$-rays produced by $7-\mathrm{GeV}$ electrons. The main electron beam is contained within the storage ring, which is twothirds of a mile in circumference. Sixteen rf cavities maintain the electron beam energy in the APS. A tuner piston in each cavity can be extended or retracted to match the cavity's resonance to the required frequency. Higher-order microwave energy penetrates the gap between the piston and sleeve, which leads to elevated temperatures if the surfaces are unprepared. The heat is a result of the secondary electrons produced from the surfaces contacting the high field of the microwaves. Multipactoring occurs when the secondary electron yield is greater than unity and can lead to problems of elevated temperatures and pressure spikes. When the surface is coated with certain materials, the number of electrons produced is lowered with the goal to reach unity. This report contains information about a number of surface coatings and their elevant properties.

\section{SET-UP}

The data in this report are from samples that range from oxygen-free high-conductivity (OFHC) copper to copper with a variety of coatings. One sample has only a titanium coating and the other two have TiN coatings. All the copper substrates are cleaned in Citranox detergent with ultrasonic agitation. The samples were rinsed in deionized water and dried in an air oven at $90{ }^{\circ} \mathrm{C}$ for 14 hours. There were two samples for each run. One was used for surface analysis and the other had its surface polished to a $3-\mu$-inch finish for measurement of film thickness.

\footnotetext{
* Work supported by the U.S. Department of Energy, Office of Basic Energy Sciences under Contract No. W-31-109-ENG-38.

†drw@aps.anl.gov
}

The variations in the coatings were brought about by changes of the film process variables. By changing the substrate temperature, substrate bias, and pulse frequency of the deposition, the composition could be manipulated. The value used in this paper to describe the composition of the TiN film is the ratio of nitrogen to titanium atoms. When the N/Ti ratio is 1.0 the film is composed of stoichiometric TiN, ratio 1:1. This does not entail all the constituents of the film. As will be shown below, the films contain other impurities, such as carbon and oxygen. The emphasis in this report is on the state of the titanium nitride and not on how the impurities affect the results; that will be addressed in future work.

After coating, the samples were inserted into the analysis system for characterization. The analysis system operates with a base pressure of $2 \times 10^{-10}$ Torr. The samples were also subjected to heat treatment of $250{ }^{\circ} \mathrm{C}$ in a vacuum of $10^{-9}$ Torr. The coatings were characterized at three different stages: the sample in the "as-received" condition, after heating, and after sputtering. At each stage, the composition of the surface was analyzed along with the secondary electron yield.

Sample heating was accomplished by passing current for 2-3 hours through a $\mathrm{W}$ filament located behind the sample. A thermocouple measured the surface temperature. After heating, the surface was further cleaned by sputtering using $\mathrm{Ar}^{+}$ions. The ions had a kinetic energy of $1.5 \mathrm{keV}$ at current of $\sim 1.5 \mu \mathrm{A}$. The samples were sputtered for $15 \mathrm{~min}$ and the ion-incident angle was 60 degrees to the sample surface normal.

\section{METHODS}

The sample was coated by mounting it onto a holder connected to the biasing power supply. Behind the sample holder, but without making contact, is a quartz halogen lamp that radiantly heats the sample so that the temperature can be independently controlled. The sample faces the magnetron-sputtering gun that holds the titanium target. Dissociating gaseous nitrogen in the sputtering gun glow discharge provides atomic nitrogen to make titanium nitride [1]. An improved power supply, which has the ability to vary the pulse frequency of the DC power feeding the gun, is employed for this set of tests. This feature increases the efficiency of the dissociation and reduces the difficulty of making TiN. The addition of heat also changes the chemistry on the sample surface and reduces the amount of impurities in the film. All the coatings reported here have a thickness of $120 \mathrm{~nm} \pm 10$ $\mathrm{nm}$ as was determined using a surface profiler. 
Surface compositions were determined by both x-ray photoelectron spectroscopy (XPS) and Auger electron spectroscopy (AES) (see Fig. 1). For AES data collection, the electron energy was set to $3 \mathrm{keV}$; for the XPS data collection, the $\mathrm{Mg} \mathrm{K} \alpha$ radiation $(1253.6 \mathrm{eV})$ emitted from a $\mathrm{Mg}$ target was used to excite the sample surfaces. A hemispheric mirror electron energy analyzer was used to collect emitted photoelectrons or Auger electrons.

The secondary electron yield (SEY) properties of the surfaces were measured using a retarding potential method [2]. The electron beam current, $I_{e}$, was determined using a Faraday cup. Electron current density of $3 \times 10^{-4}$ $\mathrm{A} / \mathrm{cm}^{2}$ was used. The SEY was measured as a function of impact electron energy, calculated by $\left(1-I_{s} / I_{e}\right)$. The sample current, $I_{s}$, was recorded by an electrometer.

\section{RESULTS AND DISCUSSION}

XPS data are shown for the sample with a N/Ti ratio of $99 \%$ (Fig. 1). Figure 1a represents the surface exposed to air for a few days after coating, which is a typical as-received condition for accelerator applications. As expected, carbon and oxygen are the major surface contaminants. To remove these contaminants, a heat treatment of $250{ }^{\circ} \mathrm{C}$ was performed. Figure $1 \mathrm{~b}$ shows the surface after 2 hours of heating and the real TiN surface is well recovered. Because the coating on this sample is formed at $>200{ }^{\circ} \mathrm{C}$ the competition between nitride and oxide formation is strongly pushed to form the nitride (due to the chemical thermodynamics of nitrogen and oxygen forming compounds with titanium). Taking into account the spectrometer sensitivity factors for various photoemission peaks, the estimated amount of oxygen in the film is $10 \%$. The ion-sputtered surface retains a similar amount of oxygen.

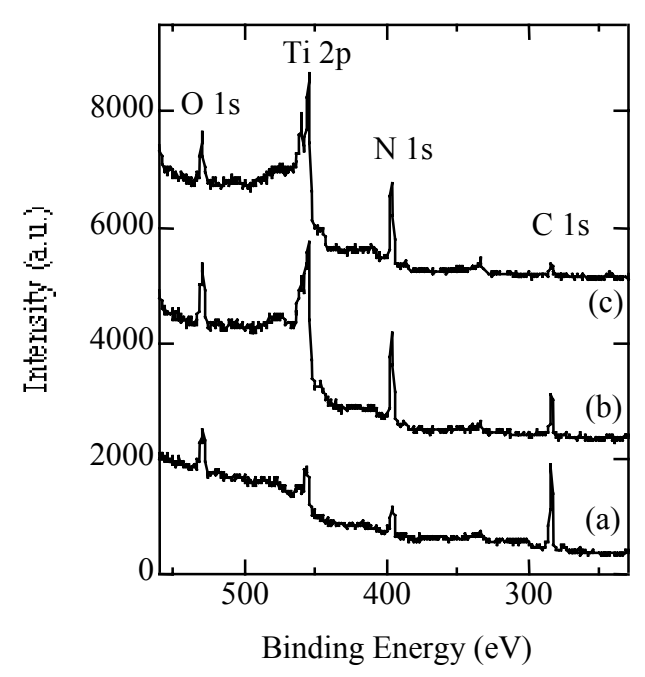

Figure 1: XPS spectra of TiN surface.

Figure 2 shows the SEY curves from 99\% N/Ti after different surface treatments. For the surface exposed to air (i.e., the as-received surface), the SEY value is 2.35 with its maximum at $290 \mathrm{eV}$ (Fig. 2a). With heat treatment, the SEY decreases significantly to 1.25 (Fig. 2b) at its maximum. The sputtered TiN surface has the smallest SEY, 1.08 (Fig. 2c).

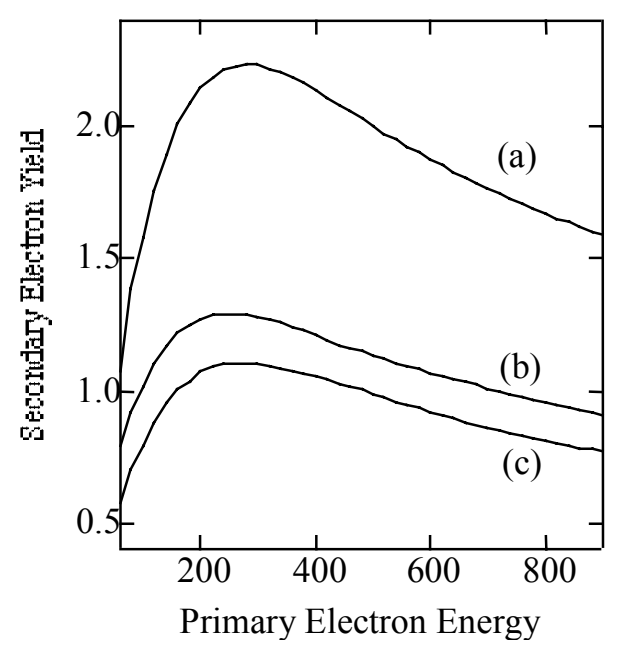

Figure 2: SEY of TiN surface.

Figure 3 is a histogram showing SEY for the different samples in this test. The "as-received" samples all show SEY values between 2.0 and 2.5. When the samples were heated all the samples improved, but the copper improved the least. The samples then had the surface sputtered to remove another level of surface contaminants and the results improved to SEYs of $\sim 1.08$, except for copper, which was 1.4. It is interesting to note that the SEY of titanium sample was just as low as those of the TiN samples (the error of the SEY measurements is $\pm 10 \%$ ).

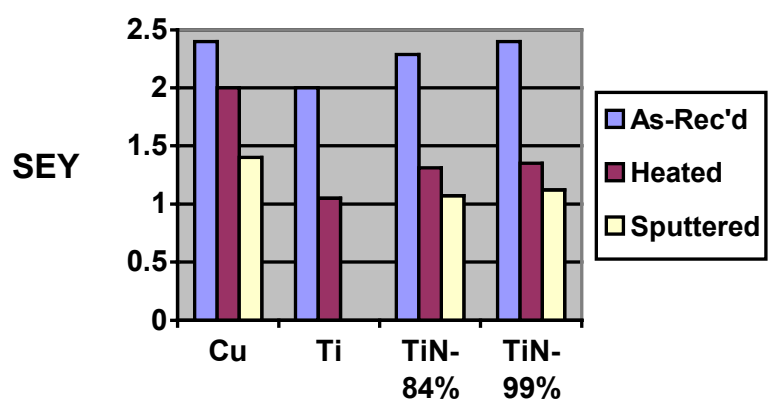

Figure 3: Histogram of SEY of copper, titanium, and titanium nitride samples.

\section{CONCLUSIONS \& FURTHER WORK}

The data reported here support the conclusion that for films within an N/Ti ratio $>80 \%$ the SEY will be $\sim 1$ after heat treatment. Such films are beneficial for the control of multipactoring. The data here are consistent with those reported in reference [3], where copper has a value of 1.3 (reported here at 1.4), and titanium is 0.9 (here, 1.05).

It remains to be determined what surface modifications if any will result from $\mathrm{rf}$ conditioning in the cavity. Is the 
conditioning more or less aggressive than the sputtering that was used in this study? The titanium coatings show very good results; however, titanium is chemically active and forms compounds with the residual gases in the cavity. The low SEY will only be achieved when the surface is clean, and will rise over time as compounds form on its surface. On the other hand, titanium nitride is chemically stable because it has saturated bonds. Once the TiN surface has been cleaned it will retain its native properties, irrespective of residual gases.

The next step for this work is to more fully determine the composition of the TiN samples. The surface morphology of the coating needs to be characterized. The effects of morphology and composition on SEY must be measured. Another characteristic to be studied is the amount of oxygen in the film. All the films have about $10 \%$ oxygen content, but this impurity has not been studied in a controlled manner. Carbon will also be studied in the future because some of its compounds have very low SEYs.

\section{ACKNOWLEDGMENTS}

The authors thank Guy Harris and Mike McDowell for their efforts in sample preparation and sample coating. We also thank George Goeppner, Richard Rosenberg, and John Noonan for their continued support for this ongoing work.

\section{REFERENCES}

[1] G. Harris, Q. Ma, D. Walters "Coating For The APS RF Cavity Tuners For The Reduction of Secondary Electrons," Proceedings of the 1999 Particle Accelerator Conference, New York, pp. 1342-1344 (1999).

[2] E.L. Garwin, et al., J. Appl. Phys. 61, 1145 (1987).

[3] Handbook of Chemistry and Physics, (R.C. Weast and M. J. Astle, eds.), CRC Press Inc. (1980). 